\title{
SPIN STRUCTURES AND CODIMENSION-TWO HOMEOMORPHISM EXTENSIONS
}

\author{
Fan Ding, Yi LiU, Shicheng Wang and Jiangang YaO
}

\begin{abstract}
Let $\imath: M \hookrightarrow \mathbb{R}^{p+2}$ be a smooth embedding from a connected, oriented, closed $p$-dimesional smooth manifold to $\mathbb{R}^{p+2}$, then there is a spin structure $\imath^{\sharp}\left(\varsigma^{p+2}\right)$ on $M$ canonically induced from the embedding. If an orientation-preserving diffeomorphism $\tau$ of $M$ extends over $\imath$ as an orientation-preserving topological homeomorphism of $\mathbb{R}^{p+2}$, then $\tau$ preserves the induced spin structure.

For $\mathcal{C}$ being Top, PL or Diff, let $\mathscr{E}_{\mathcal{C}}(\imath)$ be the subgroup of the $\mathcal{C}$-mapping class group $\operatorname{MCG}_{\mathcal{C}}(M)$ consisting of elements whose representatives extend over $\mathbb{R}^{p+2}$ as orientation-preserving $\mathcal{C}$-homeomorphisms. We apply the invariance of $\imath^{\sharp}\left(\varsigma^{p+2}\right)$ to study $\left[\operatorname{MCG}_{\mathcal{C}}(M): \mathscr{E}_{\mathcal{C}}(\imath)\right]$ when $M$ is a $p$-dimensional torus or a closed-orientable surface.
\end{abstract}

\section{Introduction}

Let $M$ be a connected, oriented, closed $p$-dimensional smooth manifold, and $\imath$ : $M \hookrightarrow \mathbb{R}^{p+2}$ be a smooth embedding. We are concerned with the question: 'how many mapping classes of $M$ extend over $\mathbb{R}^{p+2}$ ?' Regarding to different possible flavors of this question, we shall write Top (resp. PL, or Diff) for the category of topological (resp. PL, or smooth) manifolds with continuous (resp. PL or smooth) maps as morphisms, and generally write $\mathcal{C}$ for any of these categories. We speak of $\mathcal{C}$-manifolds, $\mathcal{C}$-homeomorphisms, $\mathcal{C}$-isotopies, etc., in the usual sense.

With notations above, denote $\operatorname{MCG}_{\mathcal{C}}(M)=\pi_{0} \operatorname{Homeo}_{\mathcal{C}}^{+}(M)$ for the $\mathcal{C}$-mappingclass-group of $M$, i.e., the group of $\mathcal{C}$-isotopy classes of orientation-preserving $\mathcal{C}$-selfhomeomorphisms on $M$. A class $[\tau] \in \operatorname{MCG}_{\mathcal{C}}(M)$ is called $\mathcal{C}$-extendable over $\imath$ if for some (hence any, cf. Lemma 2.2) representative $\tau$, there is an orientation-preserving $\mathcal{C}$-self-homeomorphism $\tilde{\tau}$ of $\mathbb{R}^{p+2}$ such that $\imath \circ \tau=\tilde{\tau} \circ \imath$. We define the $\mathcal{C}$-extendable subgroup with respect to $\imath$ as:

$$
\mathscr{E}_{\mathcal{C}}(\imath)=\left\{[\tau] \in \operatorname{MCG}_{\mathcal{C}}(M) \mid \tau \text { is } \mathcal{C} \text {-extendable over } \iota .\right\} .
$$

Now the question makes sense by asking what is the index of $\mathscr{E}_{\mathcal{C}}(\imath) \leq \operatorname{MCG}_{\mathcal{C}}(M)$. Note in the smooth category, as our target space is $\mathbb{R}^{p+2}$, an orientation-preserving diffeomorphism $\tau$ is diffeomorphically extendable if and only if $\imath \circ \tau$ is diffeotopic to $\imath$, (Lemma 2.3). Therefore, the question in this case is the same as counting diffeotopy classes of smooth embeddings $M \hookrightarrow \mathbb{R}^{p+2}$, which has the same image as $\imath$. Let $\varsigma^{p+2}$ be the canonical spin structure on $\mathbb{R}^{p+2}$. For any smooth embedding $\imath: M \hookrightarrow \mathbb{R}^{p+2}$, there is a canonically induced spin structure $\imath^{\sharp}\left(\varsigma^{p+2}\right)$ on $M$ (Definition 2.1). In this paper, we prove the following criterion:

Received by the editors August 3, 2011.

1991 Mathematics Subject Classification. 57N35. 
Proposition 1.1. For any smooth embedding $\imath: M \hookrightarrow \mathbb{R}^{p+2}$, the induced spin structure $\imath^{\sharp}\left(\varsigma^{p+2}\right)$ on $M$ is null spin-cobordant. Moreover, it is invariant under any orientation-preserving self-diffeomorphism of $M$ that extends over $\imath$ as an orientationpreserving topological self-homeomorphism of $\mathbb{R}^{p+2}$.

In fact, $\imath^{\sharp}\left(\varsigma^{p+2}\right)$ is naturally induced as the boundary of a spin structure on a smooth Seifert hypersurface $\Sigma$ of $\imath(M)$. Proposition 1.1 allows us to find non-trivial lower bounds of $\left[\operatorname{MCG}_{\mathcal{C}}(M): \mathscr{E}_{\mathcal{C}}(\imath)\right]$ in certain cases. In this paper, we apply the criterion to certain smoothly embedded $p$-dimensional tori in $\mathbb{R}^{p+2}$ and smoothly embedded surfaces in $\mathbb{R}^{4}$.

Denote the standard $p$-dimensional smooth torus $S^{1} \times \cdots \times S^{1}(p$ copies $)$ as $T^{p}$. The structure of $\mathrm{MCG}_{\mathcal{C}}\left(T^{p}\right)$ is fairly well understood except for $p=4$, (see Section 3 for details). This allows us to obtain the following result:

Theorem 1.1. For $p \geq 1$, suppose $\imath: T^{p} \hookrightarrow \mathbb{R}^{p+2}$ is a smooth embedding whose induced spin structure $\imath^{\sharp}\left(\varsigma^{p+2}\right)$ on $T^{p}$ is not the Lie-group spin structure, then:

$$
\left[\mathrm{MCG}_{\mathrm{Top}}\left(T^{p}\right): \mathscr{E}_{\mathrm{Top}}(\imath)\right] \geq 2^{p}-1 .
$$

Moreover, the lower bound is realized by unknotted embeddings (Definition 3.1).

Corollary 1.1. If $\imath: T^{p} \hookrightarrow \mathbb{R}^{p+2}$ is an unknotted embedding, then $\left[\mathrm{MCG}_{\text {Diff }}\left(T^{p}\right)\right.$ : $\left.\mathscr{E}_{\mathrm{Diff}}(\imath)\right]$ and $\left[\mathrm{MCG}_{\mathrm{PL}}\left(T^{p}\right): \mathscr{E}_{\mathrm{PL}}(\imath)\right]$ are finite but at least $2^{p}-1$.

The same lower bound under the assumption of Theorem 1.1 holds in the PL and the smooth categories (cf. Lemma 3.1), but it is still an interesting problem to figure out the exact bound for unknotted embeddings in both of these cases. When $p$ equals 3, an equivalent condition to the assumption of Theorem 1.1 is that a (hence any) smooth Seifert hypersurface $\Sigma^{4}$ of the knotted three-torus has signature 0 modulo 16. However, there are examples in which this condition does not hold [19]. We make the following conjecture:

Conjecture 1.1. For any smooth embedding $\imath: T^{p} \hookrightarrow \mathbb{R}^{p+2}$, $\mathscr{E}_{\mathrm{Top}}(\imath)$ is a proper subgroup of $\mathrm{MCG}_{\mathrm{Top}}\left(T^{p}\right)$.

For any integer $g>0$, denote the closed-orientable surface of genus $g$ as $F_{g}$.

Theorem 1.2. For any smooth embedding $\imath: F_{g} \hookrightarrow \mathbb{R}^{4}$, where $g>0$,

$$
\left[\mathrm{MCG}_{\mathrm{Top}}\left(F_{g}\right): \mathscr{E}_{\mathrm{Top}}(\imath)\right] \geq 2^{2 g-1}+2^{g-1} .
$$

This strengthens a result previously known for the smooth category, ([8], cf. also [17] for $g=1$ ), using the Rokhlin quadratic form [18]. The lower bound has been realized for unknotted embeddings, namely, which bounds a smoothly embedded handlebody of genus $g$ in $\mathbb{R}^{4}$, by an explicit construction of Susumu Hirose [8]. Following from the proof of Theorem 1.2, we also have an interesting corollary:

Corollary 1.2. For any $g \geq 1$, there exists $[\tau] \in \mathrm{MCG}_{\mathrm{Top}}\left(F_{g}\right)$, which is not homeomorphically extendable over any smooth embedding $\imath: F_{g} \hookrightarrow \mathbb{R}^{4}$.

In Section 2, we introduce $\imath^{\sharp}\left(\varsigma^{p+2}\right)$ (Definition 2.1) using Seifert hypersurfaces, and prove Proposition 1.1. In Section 3, we consider embedded $T^{p}$ in $\mathbb{R}^{p+2}$. We prove Theorem 1.1 and Corollary 1.1 by studying the action of $\operatorname{MCG}_{\mathcal{C}}\left(T^{p}\right)$ on $\mathcal{S}\left(T^{p}\right)$. In Section 4 , we consider embedded surfaces in $\mathbb{R}^{4}$ and using the action of $\operatorname{MCG}_{\mathcal{C}}\left(F_{g}\right)$ on the space of spin structures $\mathcal{S}\left(F_{g}\right)$ on $F_{g}$ to prove Theorem 1.2 and Corollary 1.2. 


\section{Invariant-induced spin structure}

In this section, we introduce the induced spin structures for codimension-2 closed oriented smooth submanifolds of $\mathbb{R}^{p+2}$, and prove Proposition 1.1.

2.1. Induced spin structure. In this subsection, we introduce the induced spin structures for closed oriented codimension- 2 smooth submanifolds of $\mathbb{R}^{p+2}$. We often regard a spin structure of a finite-dimensional real vector bundle over a $\mathrm{CW}$ complex as a (stabilized) trivialization over the two-skeleton up to homotopy. A spin structure of a smooth manifold is a spin structure of its tangent bundle with respect to some (hence any) CW complex structure on the manifold, which exists if and only if $M$ is orientable, and the second Stiefel-Whitney class $w_{2}(M)=0$, (cf. [12, Chapter IV], [15]).

Suppose $\imath: M \hookrightarrow \mathbb{R}^{p+2}$ is a connected, closed, oriented $p$-dimensional smooth submanifold of $\mathbb{R}^{p+2}, p \geq 1$. Since any closed oriented smooth submanifold of $\mathbb{R}^{p+2}$ has trivial Euler class ( $\left[16\right.$, Corollary 11.4]), the normal bundle of $M$ in $\mathbb{R}^{p+2}$ is trivial as $M$ is codimension 2 . On the other hand, it is well known that there exists a Seifert hypersurface:

$$
\Sigma \subset \mathbb{R}^{p+2}
$$

of $\imath(M)$, namely, a compact connected oriented $(p+1)$-dimensional smooth submanifold such that $\partial \Sigma=\imath(M)$, (cf. for example, [2, Lemma 2.2]).

Let $W$ be an inward normal vector field of $\imath(M)$ in $\Sigma$ (with respect to any compatible Riemannian metric on a collar), and $H$ be a normal vector field of $\Sigma$ in $\mathbb{R}^{p+2}$ over $\imath(M)$, such that the orientation $(W, H)$ of the normal bundle $N_{\mathbb{R}^{p+2}}(\imath(M))$ and the orientation of $M$ match up to that the canonical orientation of $\mathbb{R}^{p+2}$. The trivialization:

$$
(W, H)
$$

of $N_{\mathbb{R}^{p+2}}(\imath(M))$ defines a spin structure $\sigma$ of $N_{\mathbb{R}^{p+2}}(\imath(M))$. Recall that if $\xi=\xi^{\prime} \oplus \xi^{\prime \prime}$ is the Whitney sum of vector bundles over a CW complex, then spin structures on any two of $\xi, \xi^{\prime}$ and $\xi^{\prime \prime}$ naturally determine a spin structure of the third, so that as trivializations $\sigma \simeq \sigma^{\prime} \oplus \sigma^{\prime \prime}$ over the one-skeleton, (hence also over the two-skeleton), (cf. [12, p. 33]). Note the canonical spin structure $\varsigma^{p+2}$ of $\mathbb{R}^{p+2}$ restricts to a spin structure on $\left.T \mathbb{R}^{p+2}\right|_{\imath(M)}$. Since $\left.T \mathbb{R}^{p+2}\right|_{\imath(M)}=\imath_{*}(T M) \oplus N_{\mathbb{R}^{p+2}}(\imath(M))$, there is a complementary spin structure $\sigma^{\perp}$ of $\imath_{*}(T M)$ such that:

$$
\sigma^{\perp} \oplus \sigma=\varsigma^{p+2} \text {, }
$$

in the sense that as trivializations $\sigma^{\perp} \oplus \sigma \simeq \varsigma^{p+2}$ over the one-skeleton $M^{(1)}$.

Lemma 2.1. The spin structure $\sigma^{\perp}$ on $\imath(M)$ is independent of the choice of $\Sigma$ and $(W, H)$.

Proof. It suffices to show $\sigma$ is independent of the choice of $\Sigma$ and $(W, H)$. In fact, we show for any two choices $\Sigma,(W, H)$ and $\Sigma^{\prime},\left(W^{\prime}, H^{\prime}\right)$, the trivializations $(W, H) \simeq$ $\left(W^{\prime}, H^{\prime}\right)$ over $\imath(M)$.

First observe that any loop $\alpha$ on $\imath(M)$, when pushed into $\Sigma^{\circ}$ along $W$, becomes null-homologous in $\mathbb{R}^{p+2} \backslash \imath(M)$. To see this, consider the map $f: \mathbb{R}^{p+2} \backslash \imath(M) \rightarrow S^{1}$ defined as follows: take a tubular neighborhood $\mathcal{N}\left(\Sigma^{\circ}\right)$, where $\Sigma^{\circ}$ is the interior of $\Sigma$, and let $f \mid: \mathcal{N}(\stackrel{\circ}{\Sigma}) \rightarrow S^{1}$ to be the composition: $\mathcal{N}(\stackrel{\circ}{\Sigma}) \cong \stackrel{\circ}{\Sigma} \times I \stackrel{p}{\longrightarrow} I \stackrel{q}{\longrightarrow} I / \partial I \cong S^{1}$, 
where $p$ is the second-factor projection and $q$ is the quotient map; then extend $f \mid$ to $f: \mathbb{R}^{p+2} \backslash \imath(M) \rightarrow S^{1}$ by the constant map. Then $f_{*}: H_{1}\left(\mathbb{R}^{p+2} \backslash \imath(M)\right) \rightarrow H_{1}\left(S^{1}\right)$ is isomorphic, but the push-off of $\alpha$ along $W$ is mapped to $0 \in H_{1}\left(S^{1}\right)$, so it is null-homologous in $\mathbb{R}^{p+2} \backslash \imath(M)$.

Now $(W, H)$ and $\left(W^{\prime}, H^{\prime}\right)$ differ pointwisely by an element of $\mathrm{GL}^{+}(2, \mathbb{R})$, namely for any $x \in M,\left.\left(W^{\prime}, H^{\prime}\right)\right|_{x}=\left.(W, H)\right|_{x} \cdot R(x)$ for some $R(x) \in \mathrm{GL}^{+}(2, \mathbb{R})$. This gives a map $R: M \rightarrow \mathrm{GL}^{+}(2, \mathbb{R})$. If for some loop $\alpha: S^{1} \rightarrow M, R \circ \alpha$ were not null-homotopic in $\mathrm{GL}^{+}(2, \mathbb{R})$, then the push-offs of $\alpha$ along $W$ and $W^{\prime}$ would differ by a non-zero multiple of the meridian $\mu$, namely the loop that bounds a normal disk of $\imath(M)$. Since $\mu$ is the generator of $H_{1}\left(\mathbb{R}^{p+2} \backslash \imath(M)\right) \cong \mathbb{Z}$ by the Alexander duality, the two push-offs would not be both null-homologous in $\mathbb{R}^{p+2} \backslash \imath(M)$, which is a contradiction. Thus $R_{\sharp}: \pi_{1}(M) \rightarrow \pi_{1}\left(\mathrm{GL}^{+}(2, \mathbb{R})\right)$ is trivial. We conclude that $R$ is homotopic to the constant identity map as $\pi_{i}\left(\mathrm{GL}^{+}(2, \mathbb{R})\right) \cong \pi_{i}\left(S^{1}\right)$ is trivial for $i \geq 2$. This implies $(W, H) \simeq\left(W^{\prime}, H^{\prime}\right)$ over $M$.

Lemma 2.1 allows us to make the following definition.

Definition 2.1. For a smooth embedding $\imath: M \hookrightarrow \mathbb{R}^{p+2}$ of a connected, closed, oriented $p$-dimensional smooth manifold $M$ into $\mathbb{R}^{p+2}$, we define the induced spin structure as:

$$
\imath^{\sharp}\left(\varsigma^{p+2}\right)=\imath^{*}\left(\sigma^{\perp}\right),
$$

where $\sigma^{\perp}$ is as described above.

2.2. Homeomorphism extensions. In this subsection, we prove Proposition 1.1. Recall that for a spin manifold $M$ with boundary $\partial M, \partial M$ has a natural spin structure induced from the spin structure of $M$ and the (inward) normal vector of $\partial M$ in $M$. A manifold is said to be null spin-cobordant if there is a spin manifold bounded by it, inducing its spin structure, (cf. [12, Chapter IV], [15]).

Proof of Proposition 1.1. We first show $\imath^{\sharp}\left(\varsigma^{p+2}\right)$ is null spin-cobordant, or equivalently that $\sigma^{\perp}$ is a spin boundary. In fact, for a Seifert hypersurface $\Sigma$ of $\imath(M)$, the normal vector field $H$ of $\Sigma$ in $\mathbb{R}^{p+2}$ defines a spin structure $\sigma_{H}$ on the normal bundle $N_{\mathbb{R}^{p+2}}(\Sigma)$, so there is a spin structure $\sigma_{H}^{\perp}$ on $T \Sigma$ such that:

$$
\sigma_{H}^{\perp} \oplus \sigma_{H}=\varsigma^{p+2},
$$

on $T \Sigma \oplus N_{\mathbb{R}^{p+2}}(\Sigma)=\left.T \mathbb{R}^{p+2}\right|_{\Sigma}$. The spin boundary of $\left(\Sigma, \sigma_{H}^{\perp}\right)$ is clearly $\left(M, \sigma^{\perp}\right)$ by the construction.

We next prove the invariance of $\imath^{\sharp}\left(\varsigma^{p+2}\right)$ under homeomorphically extendable selfdiffeomorphisms. Specifically, for an orientation-preserving self-diffeomorphism $\tau$ : $M \rightarrow M$, which extends over $\imath$ as an orientation-preserving self-homeomorphism $\tilde{\tau}$ of $\mathbb{R}^{p+2}$, we must show $\imath^{\sharp}\left(\varsigma^{p+2}\right)$ equals $\tau^{*}\left(\imath^{\sharp}\left(\varsigma^{p+2}\right)\right)=(\imath \circ \tau)^{\sharp}\left(\varsigma^{p+2}\right)$. Without loss of generality, we may assume $p>1$ as there is nothing to prove for $p=1$. We shall omit writing $\imath$ identifying $M$ as a submanifold of $\mathbb{R}^{p+2}$, and identify $D^{2}$ as the unit disk in $\mathbb{C}$.

Let $\mathcal{N}$ be a closed tubular neighborhood of $M$ in $\mathbb{R}^{p+2}$, identified with $M \times D^{2}$ such that $M$ is identified with $M \times\{0\}$ and $M \times\{1\}$ is the push-off of $M$ along $W$. By the uniqueness of normal bundle for codimension 2 locally flat embedding (see [13] for the case of the ambient dimension at least 5, and [3, Section 9.3] for the 
ambient dimension equal to 4), we may assume $\tilde{\tau}$ preserves $\mathcal{N}$, and restricted to this neighborhood, it is a bundle map:

$$
\tilde{\tau}: M \times D^{2} \rightarrow M \times D^{2},
$$

given by $\tilde{\tau}(x, v) \rightarrow(\tau(x), R(x) \cdot v)$, where $R(x) \in \mathrm{SO}(2)$.

Since $\tilde{\tau}(\Sigma)$ is still a (topological) Seifert hypersurface, by the same argument of Lemma 2.1, $R: M \rightarrow \mathrm{SO}(2)$ is homotopic to the constant identity map. This implies that $\left.\tilde{\tau}\right|_{N}$ may be assumed to be $\tau \times \operatorname{id}_{D^{2}}$ under the identification $\mathcal{N} \cong M \times D^{2}$. Let:

$$
X=S^{p+2} \backslash \mathcal{N}
$$

where $S^{p+2}=\mathbb{R}^{p+2} \cup\{\infty\}$. Extend $\tilde{\tau}$ to a homeomorphism of $S^{p+2}$, still denoted as $\tilde{\tau}$, by defining $\tilde{\tau}(\infty)=\infty$. We glue two (opposite) copies $X,-X$ along the boundary via $\left.\tilde{\tau}\right|_{\partial X}: \partial X \rightarrow \partial X$, and the resulting smooth manifold is denoted as:

$$
Y_{\tau}=X \cup_{\tau}(-X)
$$

On the other hand, we may glue via id $\left.\right|_{\partial X}$ to obtain the double of $X$, denoted as:

$$
Y_{\mathrm{id}}=X \cup_{\mathrm{id}}(-X) \text {. }
$$

Thus $Y_{\tau}$ is homeomorphic to $Y_{\text {id }}$ via $\tilde{\tau} \cup$ id.

Observe that $T Y_{\text {id }}$ is stably trivial. In fact, $X \subset S^{p+2}=\partial D^{p+3}$, and we may push the interior of $X$ into the interior of $D^{p+3}$ so that $(X, \partial X) \subset\left(D^{p+3}, S^{p+2}\right)$ is a proper embedding of pairs. We may further assume that on the collar neighborhood of $\partial D^{p+3}$, diffeomorphically $S^{p+2} \times I, X$ is identified as $\partial X \times I$. Then doubling $D^{p+3}$ along boundary gives a codimension 1 smooth embedding $Y_{\text {id }} \subset S^{p+3}$. Hence, clearly $T Y_{\text {id }} \oplus \epsilon^{1}$ is trivial, so $w_{2}\left(Y_{\text {id }}\right)=0$ in $H^{2}\left(Y_{\text {id }} ; \mathbb{Z}_{2}\right)$, or in other words, $Y_{\text {id }}$ is spin.

We wish to show, however, that $Y_{\tau}$ is not spin if $\tau$ does not preserve $\imath^{\sharp}\left(\varsigma^{p+2}\right)$. This would lead to a contradiction because an orientable smooth manifold is spin if and only if its second Stiefel-Whitney class vanishes, and because the Stiefel-Whitney class depends only on the homotopy type of the smooth manifold, (cf. [20], also [16]). Recall also that for an oriented smooth manifold $M$ which admits spin structures, the space $\mathcal{S}(M)$ of spin structures on $M$, is an affine $H^{1}\left(M ; \mathbb{Z}_{2}\right)$. Thus any two spin structures $\sigma_{1}, \sigma_{0} \in \mathcal{S}(M)$ gives rise to a natural difference homomorphism:

$$
\sigma_{1}-\sigma_{0}: \pi_{1}(M) \rightarrow \mathbb{Z}_{2}
$$

which may be regarded as an element in $H^{1}\left(M ; \mathbb{Z}_{2}\right)$. Specifically, $\sigma_{1}-\sigma_{0}$ takes the value 0 (resp. 1) in $\mathbb{Z}_{2}$ at $[\alpha] \in \pi_{1}(M)$, if $\sigma_{1}$ and $\sigma_{0}$ are represented by the same trivialization (resp. two distinct trivializations) of $T M$ over the loop $\alpha$ up to homotopy.

Suppose $\tau$ did not preserve $\imath^{\sharp}\left(\varsigma^{p+2}\right)$. Then there is some smoothly embedded loop $\alpha \subset M$ such that $\left.\left.\imath^{\sharp}\left(\varsigma^{p+2}\right)\right|_{\alpha} \nsucceq \tau^{*}\left(\imath^{\sharp}\left(\varsigma^{p+2}\right)\right)\right|_{\alpha}$. Since we assumed that $\left.\tilde{\tau}\right|_{\mathcal{N}}=\tau \times$ $\operatorname{id}_{D^{2}}$ under the identification $\mathcal{N} \cong M \times D^{2}$, by the construction of $\imath^{\sharp}\left(\varsigma^{p+2}\right)$, the difference $\tilde{\tau}_{*}\left(\left.\varsigma^{p+2}\right|_{\mathcal{N}}\right)-\left.\varsigma^{p+2}\right|_{\mathcal{N}}$ in $H^{1}\left(\mathcal{N} ; \mathbb{Z}_{2}\right)$ is exactly $\tau_{*}\left(\imath^{\sharp}\left(\varsigma^{p+2}\right)\right)-\left(\imath^{\sharp}\left(\varsigma^{p+2}\right)\right)$ in $H^{1}\left(M ; \mathbb{Z}_{2}\right)$, under the natural isomorphism $H^{1}\left(\mathcal{N} ; \mathbb{Z}_{2}\right) \rightarrow H^{1}\left(M ; \mathbb{Z}_{2}\right)$ via inclusion. Hence, $\left.\tilde{\tau}_{*}\left(\left.\varsigma^{p+2}\right|_{\alpha \times\{1\}}\right) \not \varsigma^{p+2}\right|_{\tau(\alpha) \times\{1\}}$.

As $\alpha \times\{1\}$ is null-homological in $X$ by the construction (cf. the proof of Lemma 2.1), it bounds a smoothly immersed oriented surface $j: F \leftrightarrow X$ such that $j(\stackrel{\circ}{F}) \subset \stackrel{\circ}{X}$, and $j$ is a smooth embedding in a collar neighborhood of $\partial F$. This can be seen by writing $\alpha$ as a product of commutators, so there is a continuous map $F \rightarrow X$, which can 
be perturbed to be an immersion by the Whitney's trick. Thus, there is a smoothly immersed closed oriented surface:

$$
\widehat{j}: K=F \cup(-F) \rightarrow Y_{\tau},
$$

defined by $j \cup(-\tilde{\tau} \circ j)$. Note $\left.\widehat{j}^{*}\left(T Y_{\tau}\right)\right|_{F}=\left.j^{*}\left(T \mathbb{R}^{p+2}\right)\right|_{F}$ has a spin structure $\left.\varsigma^{p+2}\right|_{F}$, and $\left.\widehat{j}^{*}\left(T Y_{\tau}\right)\right|_{-F}=\left.(-\tilde{\tau} \circ j)^{*}\left(T \mathbb{R}^{p+2}\right)\right|_{-F}$ has a spin structure $-\left.\varsigma^{p+2}\right|_{(-\tilde{\tau}(F))}$. These two spin structures disagree along $\alpha \times\{1\} \subset \partial X$ (corresponding to $\tau(\alpha) \times\{1\} \subset \partial(-X)$ ), so implies that $w_{2}\left(\widehat{j}^{*}\left(T Y_{\tau}\right)\right) \in H^{2}\left(K ; \mathbb{Z}_{2}\right)$ does not vanish.

We conclude $w_{2}\left(Y_{\tau}\right) \in H^{2}\left(Y_{\tau} ; \mathbb{Z}_{2}\right)$ does not vanish either, as it is the pull-back of a non-trivial element $w_{2}\left(\hat{j}^{*}\left(T Y_{\tau}\right)\right) \in H^{2}\left(K ; \mathbb{Z}_{2}\right)$. In other words, $Y_{\tau}$ is not spin, which yields a contradiction as desired.

Remark 2.1. We are aware that the induced spin structure $\imath^{\sharp}\left(\varsigma^{p+2}\right)$ can also be derived from a general construction of characteristic pairs ( $[14]$, cf. also $[2,4])$. Recall that a pair of oriented compact smooth manifolds $(W, M)$ is called characteristic if $M \subset W$ is a proper codimension-2 submanifold dual to $w_{2}(M)$. The space $\operatorname{Char}(W, M)$ of characterizations of $(W, M)$ consists of spin structures on $W \backslash M$, which does not extend across any component of $M$, admitting a natural free transitive action $H^{1}\left(W ; \mathbb{Z}_{2}\right)$. There is a function $h: \operatorname{Char}(W, M) \rightarrow \mathcal{S}(M)$ equivariant under the natural actions of $H^{1}\left(W ; \mathbb{Z}_{2}\right)$ on $\operatorname{Char}(W, M)$ and $H^{1}\left(M ; \mathbb{Z}_{2}\right)$ on $\mathcal{S}(M)$ via the homomorphism $H^{1}\left(W ; \mathbb{Z}_{2}\right) \rightarrow H^{1}\left(M ; \mathbb{Z}_{2}\right)$, where $\mathcal{S}(M)$ is the space of spin structures on $M,\left(\left[14\right.\right.$, Definition 6.1, Theorem 2.4, Lemma 6.2]). When $W=S^{p+2}$ and $M$ is connected, $\operatorname{Char}(W, M)$ is a single-element group whose image under $h$ coincides with $\imath^{\sharp}\left(\varsigma^{p+2}\right)$. This gives an alternative proof of Proposition 1.1 if one assumes $\tau$ extends over $S^{p+2}$ diffeomorphically rather than just homeomorphically.

Before going to the applications, we mention the following lemma, which justifies the well-definedness of $\mathscr{E}_{\mathcal{C}}(\imath)$.

Lemma 2.2. Let $\imath: M \hookrightarrow \mathbb{R}^{p+2}$ be a smooth embedding of an orientable closed p-dimensional manifold. Let $\tau, \tau^{\prime}: M \rightarrow M$ be two $\mathcal{C}$-isotopic orientation-preserving $\mathcal{C}$-homeomorphisms, then $\tau$ is $\mathcal{C}$-extendable if and only if $\tau^{\prime}$ is $\mathcal{C}$-extendable over .

Proof. First assume $\tau^{\prime}$ is the identity. Take a tubular neighborhood $\mathcal{N}$ of $\imath(M)$ in $\mathbb{R}^{p+2}$, we have seen that $\mathcal{N}$ is diffeomorphic to $M \times D^{2}$. As $\tau$ is $\mathcal{C}$-isotopic to the identity, say $f_{t}: M \rightarrow M$ where $t \in[0,1]$, we define:

$$
\tilde{\tau} \mid: M \times D^{2} \rightarrow M \times D^{2},
$$

by $\tilde{\tau}\left(x, r e^{i \theta}\right)=\left(f_{r}(x), r e^{i \theta}\right)$, where $D^{2}$ is identified as the unit disk of $\mathbb{C}$. Then $\tilde{\tau}$ is the identity restricted to $\partial \mathcal{N} \cong M \times \partial D^{2}$. We may further extend $\tilde{\tau}$ outside $\mathcal{N}$ over $\mathbb{R}^{p+2}$ by the identity. This implies $\tau$ is $\mathcal{C}$-extendable.

In the general case, let $\tau, \tau^{\prime}$ be two orientation-preserving $\mathcal{C}$-homeomorphisms which are $\mathcal{C}$-isotopic. Then $\tau^{-1} \circ \tau^{\prime}$ is $\mathcal{C}$-isotopic to the identity, so $\mathcal{C}$-extendable. Let $\phi$ : $\mathbb{R}^{p+2} \rightarrow \mathbb{R}^{p+2}$ be an orientation-preserving $\mathcal{C}$-homeomorphic extension of $\tau^{-1} \circ \tau^{\prime}$. If $\tau$ is $\mathcal{C}$-extendable, say as $\tilde{\tau}: \mathbb{R}^{p+2} \rightarrow \mathbb{R}^{p+2}$, then $\tau^{\prime}$ may be extended as $\tilde{\tau} \circ \phi$, and vice versa.

We thank the referee for pointing out the following reformulation of extendable diffeomorphisms in the smooth category: 
Lemma 2.3. Let $\imath: M \hookrightarrow \mathbb{R}^{p+2}$ be a smooth embedding of an orientable closed p-dimensional manifold. An orientation-preserving self-diffeomorphism $\tau: M \rightarrow M$ extends over $\mathbb{R}^{p+2}$ as an orientation-preserving self-diffeomorphism if and only if $\imath \circ \tau$ is diffeotopic to $\imath$.

Proof. If $\tau$ is diffeomorphically extendable, we have $\imath \circ \tau=\tilde{\tau} \circ \imath$ for some orientationpreserving diffeomorphism $\tilde{\tau}: \mathbb{R}^{p+2} \rightarrow \mathbb{R}^{p+2}$, which is diffeotopic to the identity following from the well known Alexander trick, (cf. [9, Chapter 8, Section 3]). Thus $\imath \circ \tau$ is diffeotopic to $\imath$. On the other hand, if $\imath \circ \tau$ is diffeotopic to $\imath$, there is a diffeotopy $H_{t}: \mathbb{R}^{p+2} \rightarrow \mathbb{R}^{p+2}$, where $0 \leq t \leq 1$, such that $H_{0}$ is the identity and $H_{1}$ satisfies $H_{1} \circ \imath=\imath \circ \tau$ by the isotopy extension theorem in the smooth category, (cf. [9, Chapter 8, Theorem 1.3]). This means $H_{1}$ is an extension of $\tau$ via $\imath$.

\section{Embedded $p$-tori in $\mathbb{R}^{p+2}$}

In this section, we prove Theorem 1.1 and its corollaries. Throughout this section, we shall fix a parametrization $T^{p}=S_{1}^{1} \times \cdots \times S_{p}^{1}$ for a standard $p$-dimensional torus $T^{p}$, where each $S_{i}^{1}$ is a copy of the unit circle $S^{1} \subset \mathbb{C}$.

3.1. Index lower bounds. We start by some general facts about $\mathrm{MCG}_{\mathcal{C}}\left(T^{p}\right)$ and its action on the space $\mathcal{S}\left(T^{p}\right)$ of spin structures on $T^{p}$.

For any $p \geq 2$, Homeo ${ }_{\mathcal{C}}^{+}\left(T^{p}\right)$ has a modular subgroup $\operatorname{Mod}\left(T^{p}\right) \cong \operatorname{SL}(p, \mathbb{Z})$ generated by elements represented by the Dehn twists $\tau_{i, j}(1 \leq i, j \leq p, i \neq j)$ along the $i$ th factor in the $S_{i}^{1} \times S_{j}^{1}$ direction, defined by:

$$
\tau_{i, j}\left(u_{1}, \ldots, u_{p}\right)=\left(u_{1}, \ldots, u_{j-1}, u_{i} u_{j}, u_{j+1}, \ldots, u_{p}\right) .
$$

$\operatorname{Mod}\left(T^{p}\right)$ may be regarded as a subgroup of $\operatorname{MCG}_{\mathcal{C}}\left(T^{p}\right)$ under the natural quotient $\pi_{0}: \operatorname{Homeo}_{\mathcal{C}}^{+}\left(T^{p}\right) \rightarrow \operatorname{MCG}_{\mathcal{C}}\left(T^{p}\right)$. Thus the action of $\mathrm{MCG}_{\mathcal{C}}\left(T^{p}\right)$ on $H_{1}\left(T^{p} ; \mathbb{Z}\right)$ induces a splitting sequence of groups:

$$
1 \rightarrow \mathscr{I}_{\mathcal{C}}\left(T^{p}\right) \rightarrow \operatorname{MCG}_{\mathcal{C}}\left(T^{p}\right) \rightarrow \mathrm{SL}(p, \mathbb{Z}) \rightarrow 1
$$

as $\operatorname{Aut}^{+}\left(H_{1}\left(T^{p} ; \mathbb{Z}\right)\right) \cong \operatorname{SL}(p, \mathbb{Z})$, (which holds trivially for $p=1$ as well). In other words, $\operatorname{MCG}_{\mathcal{C}}\left(T^{p}\right)=\mathscr{I}_{\mathcal{C}}\left(T^{p}\right) \rtimes \operatorname{Mod}\left(T^{p}\right)$. It is well known that $\operatorname{MCG}_{\mathcal{C}}\left(T^{2}\right)=\operatorname{Mod}\left(T^{2}\right)$ (cf. [11]), and $\operatorname{MCG}_{\mathcal{C}}\left(T^{3}\right)=\operatorname{Mod}\left(T^{3}\right)$ follows from general results of Hatcher for Haken three-manifolds $[5,7]$. While the case $p=4$ remains mysterious, for $p \geq 5$, the splitting is known to be non-trivial and $\operatorname{MCG}_{\mathcal{C}}\left(T^{p}\right)$ are different for various $\mathcal{C}$ 's. Specifically, a theorem of Hatcher ([6, Theorem 4.1], cf. also [10]) implies $\mathscr{I}_{\mathcal{C}}\left(T^{p}\right)$ $(p \geq 5)$ is an infinitely generated abelian group, which can be regarded as a $\mathrm{SL}(p, \mathbb{Z})$ module with the following decomposition:

$$
\mathscr{I}_{\text {Diff }}\left(T^{p}\right) \cong \mathscr{W}_{p} \oplus H^{2}\left(T^{p} ; \mathbb{Z}_{2}\right) \oplus \bigoplus_{i=1}^{p} H^{i}\left(T^{p} ; \Gamma_{i+1}\right)
$$

and $\mathscr{I}_{\mathrm{PL}}\left(T^{p}\right) \cong \mathscr{W}_{p} \oplus H^{2}\left(T^{p} ; \mathbb{Z}_{2}\right), \mathscr{I}_{\mathrm{Top}}\left(T^{p}\right) \cong \mathscr{W}_{p}$ as induced by the forgetting quoients. Here $\mathscr{W}_{p} \cong \mathbb{Z}_{2}\left[t_{1}, t_{1}^{-1}, \ldots, t_{p}, t_{p}^{-1}\right] / \mathbb{Z}_{2}\left[t_{1}+t_{1}^{-1}, \ldots, t_{p}+t_{p}^{-1}\right] \cong \mathbb{Z}_{2}^{\oplus \infty}$ has the natural action induced by that of $\operatorname{SL}(p, \mathbb{Z})$ on the monomials, and $\Gamma_{i}$ is the $i$ th Kervaire-Milnor group of homotopy spheres, which is finite abelian, $i \geq 0$, and the $\mathrm{SL}(p, \mathbb{Z})$ acts on $H^{2}\left(T^{p} ; \mathbb{Z}_{2}\right), H^{i}\left(T^{p} ; \Gamma_{i+1}\right)$ naturally as usual. 
As the space of spin structures $\mathcal{S}\left(T^{p}\right)$ is an affine $H^{1}\left(T^{p} ; \mathbb{Z}_{2}\right)$, there is a Lie-group spin structure and $2^{p}-1$ non-Lie-group spin structures. Denote the subset of nonLie-group spin structures as $\mathcal{S}^{\star}\left(T^{p}\right)$.

The lower bound in Theorem 1.1 follows from the lemma below.

Lemma 3.1. $\mathrm{MCG}_{\mathcal{C}}\left(T^{p}\right)$ fixes the Lie-group spin structure of $T^{p}$, and acts transitively on $\mathcal{S}^{\star}\left(T^{p}\right)$. Hence $\left[\mathrm{MCG}_{\mathcal{C}}\left(T^{p}\right): \mathscr{E}_{\mathcal{C}}(\imath)\right] \geq 2^{p}-1$ if $\imath: T^{p} \hookrightarrow \mathbb{R}^{p+2}$ induces a non-Liegroup spin structure $\imath^{\sharp}\left(\varsigma^{p+2}\right)$ on $T^{p}$.

Proof. For the standard parametrization $u=\left(u_{1}, \ldots, u_{p}\right)$ of $T^{p}=S_{1}^{1} \times \cdots \times S_{p}^{1}$, the Lie group spin structure $\sigma_{0} \in \mathcal{S}\left(T^{p}\right)$ is represented by the standard framing $\left(\frac{\partial}{\partial u_{1}}, \ldots, \frac{\partial}{\partial u_{p}}\right)$ over $T^{p}$, so for any $\tau \in \operatorname{Mod}\left(T^{p}\right)$,

$$
\left.\tau_{*}^{-1}\left(\frac{\partial}{\partial u_{1}}, \ldots, \frac{\partial}{\partial u_{p}}\right)\right|_{u}=\left.\left(\frac{\partial}{\partial u_{1}}, \ldots, \frac{\partial}{\partial u_{p}}\right)\right|_{\tau(u)} \cdot A,
$$

for the matrix $A \in \mathrm{SL}(p, \mathbb{Z})$ defining $\tau$ for any $u \in T^{p}$. This means pulling back by $\tau$ fixes the framing over $T^{p}$ up to homotopy, so $\tau^{*}\left(\sigma_{0}\right)=\sigma_{0}$. On the other hand, $\mathscr{I}_{\mathcal{C}}\left(T^{p}\right)$ fixes $\sigma$ since the action of $\operatorname{MCG}_{\mathcal{C}}\left(T^{p}\right)$ descends to $\operatorname{Aut}^{+}\left(H_{1}\left(T^{p}\right)\right) \cong \operatorname{SL}(p, \mathbb{Z})$. Thus $\operatorname{MCG}_{\mathcal{C}}\left(T^{p}\right)$ fixes $\sigma_{0}$.

Let $\sigma^{\prime}, \sigma^{\prime \prime} \in \mathcal{S}^{\star}\left(T^{p}\right)$, the differences $\sigma^{\prime}-\sigma_{0}, \sigma^{\prime \prime}-\sigma_{0} \in H^{1}\left(T^{p} ; \mathbb{Z}_{2}\right) \backslash\{0\}$. As $\operatorname{MCG}_{\mathcal{C}}\left(T^{p}\right)$ acts transitively on $H^{1}\left(T^{p} ; \mathbb{Z}_{2}\right) \backslash\{0\}$ and fixes $\sigma_{0}$, there is some $[\tau] \in$ $\operatorname{MCG}_{\mathcal{C}}\left(T^{p}\right)$ such that $\tau^{*}\left(\sigma^{\prime}\right)=\sigma^{\prime \prime}$. Thus $\operatorname{MCG}_{\mathcal{C}}\left(T^{p}\right)$ acts transitively on $\mathcal{S}^{\star}\left(T^{p}\right)$.

Finally, by Proposition 1.1, $\tau \in \mathscr{E}_{\mathcal{C}}(\imath)$ only if $\tau$ fixes $\imath^{\sharp}\left(\varsigma^{p+2}\right)$, so the transitivity implies $\left[\operatorname{MCG}_{\mathcal{C}}\left(T^{p}\right): \mathscr{E}_{\mathcal{C}}(\imath)\right] \geq\left|\mathcal{S}^{\star}\left(T^{p}\right)\right|=2^{p}-1$ if $\imath^{\sharp}\left(\varsigma^{p+2}\right) \in \mathcal{S}^{\star}\left(T^{p}\right)$.

A little more can be said about $\mathscr{E}_{\mathcal{C}}(\imath)$ for general smooth embeddings of $T^{p}$ into $\mathbb{R}^{p+2}$.

Lemma 3.2. For $p \geq 1$, and for any smooth embedding $\imath: T^{p} \hookrightarrow \mathbb{R}^{p+2}, \mathscr{I}_{\mathcal{C}}\left(T^{p}\right) \cap$ $\mathscr{E}_{\mathcal{C}}(\imath)$ has finite index in $\mathscr{I}_{\mathcal{C}}\left(T^{p}\right)$. Moreover, $\mathscr{I}_{\text {Top }}\left(T^{p}\right) \leq \mathscr{E}_{\mathrm{Top}}(\imath)$.

Proof. Without loss of generality, we may assume $p \geq 4$ as $\mathscr{I}_{\mathcal{C}}\left(T^{p}\right)$ is trivial when $p \leq 3$.

First suppose $p \geq 5$. In this case, it suffices to show $\mathscr{W}_{p} \leq \mathscr{E}_{\text {Diff }}(\imath)$. Let $[\tau] \in \mathscr{W}_{p}$ where $\tau$ is a diffeomorphic representative. By Remark (4) of [6, Theorem 4.1], $\tau$ is smoothly concordant to id, namely, there is a diffeomorphism $f: T^{p} \times[0,1] \rightarrow$ $T^{p} \times[0,1]$, such that $\left.f\right|_{T^{p} \times\{0\}}=\tau,\left.f\right|_{T^{p} \times\{1\}}=\operatorname{id}_{T^{p}}$. Let $f_{T}, f_{I}$ be the first and the second component of $f$, respectively, i.e., such that $f(u, r)=\left(f_{T}(u, r), f_{I}(u, r)\right)$. Pick a tubular neighborhood $\mathcal{N} \cong T^{p} \times D^{2}$ of $\imath\left(T^{p}\right)$ in $\mathbb{R}^{p+2}$. Identify $D^{2}$ as the unit disk of $\mathbb{C}$, and define $\tilde{\tau} \mid: T^{p} \times D^{2} \rightarrow T^{p} \times D^{2}$ by $\tilde{\tau}\left(u, r e^{i \theta}\right)=\left(f_{T}(u, r), f_{I}(u, r) e^{i \theta}\right)$. It is clear that $\tilde{\tau} \mid$ is an orientation-preserving diffeomorphism, which restrict to $T^{p} \times \partial D^{2}$ as identity. We may define an orientation-preserving diffeomorphism $\tilde{\tau}: \mathbb{R}^{p+2} \rightarrow \mathbb{R}^{p+2}$ by extending $\tilde{\tau} \mid$ as identity outside $\mathcal{N}$, which extends $\tau$. This shows $[\tau] \in \mathscr{E}_{\text {Diff }}(l)$.

For $p=4$, let $[\tau] \in \mathscr{I}_{\mathcal{C}}\left(T^{4}\right)$ where $\tau$ is a $\mathcal{C}$-homeomorphic representative. Pick a tubular neighborhood $\mathcal{N} \cong T^{4} \times D^{2}$ of $\imath\left(T^{4}\right)$ in $\mathbb{R}^{6}$. We first define $\tilde{\tau}: T^{4} \times D^{2}\left(\frac{1}{2}\right) \rightarrow$ $T^{4} \times D^{2}\left(\frac{1}{2}\right)$ as $\tau \times \operatorname{id}_{D^{2}\left(\frac{1}{2}\right)}$, where $D^{2}\left(\frac{1}{2}\right)$ is the disk of radius one half. $\tilde{\tau} \mid$ restricted to $T^{4} \times \partial D^{2}\left(\frac{1}{2}\right)$ may be regarded as an element of $\mathscr{I}_{\mathcal{C}}\left(T^{5}\right)$. If it lies in $\mathscr{W}_{5}$, then there is a $\mathcal{C}$-concordance $f: T^{5} \times[0,1] \rightarrow T^{5} \times[0,1]$ between $\left.\tilde{\tau}\right|_{T^{4} \times \partial D^{2}\left(\frac{1}{2}\right)}$ and the 
identity obtained by joining a $\mathcal{C}$-isotopy between $\left.\tilde{\tau}\right|_{T^{4} \times \partial D^{2}\left(\frac{1}{2}\right)}$ and a diffeomorphic representative $\phi \in[\tau]$ with a smooth concordance between $\phi$ and the identity. As $T^{5} \times[0,1] \cong T^{4} \times\left(D^{2} \backslash \stackrel{\circ}{D}^{2}\left(\frac{1}{2}\right)\right)$, we may extend $\tilde{\tau} \mid$ using the $\mathcal{C}$-concordance $f$ over $\mathcal{N}$ such that $\left.\tilde{\tau}\right|_{\partial \mathcal{N}}$ is the identity. Further extend $\tilde{\tau} \mid$ outside $\mathcal{N}$ by the identity, we see $[\tau] \in \mathscr{E}_{\mathcal{C}}(\imath)$. This means the preimage of $\mathscr{W}_{5}$ under:

$$
\mathscr{I}_{\mathcal{C}}\left(T^{4}\right) \rightarrow \mathscr{I}_{\mathcal{C}}\left(T^{5}\right)
$$

defined by $[\tau] \rightarrow\left[\tau \times \mathrm{id}_{S^{1}}\right]$, is contained in $\mathscr{E}_{\mathcal{C}}(\imath)$. Since $\mathscr{W}_{5}$ has finite index in $\mathscr{I}_{\mathcal{C}}\left(T^{5}\right)$, we conclude $\mathscr{I}_{\mathcal{C}}\left(T^{4}\right) \cap \mathscr{E}_{\mathcal{C}}(\imath)$ has finite index in $\mathscr{I}_{\mathcal{C}}\left(T^{4}\right)$ as well. Moreover, $\mathscr{I}_{\text {Top }}\left(T^{4}\right) \leq$ $\mathscr{E}_{\text {Top }}(\imath)$ since $\mathscr{W}_{5}=\mathscr{I}_{\text {Top }}\left(T^{5}\right)$.

We proceed to consider unknotted embeddings of $T^{p}$ into $\mathbb{R}^{p+2}$. These have been defined and studied in [1]. We recall the notion and properties enough for our use here. Regard $S^{1}$ and $D^{2}$ as the unit circle and the unit disk of $\mathbb{C}$, respectively. The standard basis of $\mathbb{R}^{n}$ is $\left(\vec{\varepsilon}_{1}, \ldots, \vec{\varepsilon}_{n}\right)$, and the $m$-subspace spanned by $\left(\vec{\varepsilon}_{i_{1}}, \ldots, \vec{\varepsilon}_{i_{m}}\right)$ will be written as $\mathbb{R}_{i_{1}, \ldots, i_{m}}^{m}$, and hence $\mathbb{R}^{n}=\mathbb{R}_{1, \ldots, n}^{n} \subset \mathbb{R}^{n+1}$.

Example 3.1 (The standard model). Let $\imath_{0}:$ pt $=T^{0} \rightarrow \mathbb{R}^{2}$ be $\imath_{0}(\mathrm{pt})=0$ by convention. Inductively suppose $\imath_{p-1}$ has been constructed for some $p \geq 1$ such that $\imath_{p-1}\left(T^{p-1}\right) \subset \stackrel{\circ}{D}^{p} \subset \mathbb{R}_{2, \ldots, p+1}^{p}$. Denote the rotation of $\mathbb{R}^{p+2}$ on the subspace $\mathbb{R}_{2, p+2}^{2}$ of angle $\arg (u)$ as $\rho_{p}(u) \in \mathrm{SO}(p+2)$, for any $u \in S^{1}$, and we may define $\imath_{p}: T^{p}=$ $T^{p-1} \times S_{p}^{1}$ as:

$$
\imath_{p}(v, u)=\rho_{p}(u)\left(\frac{1}{2} \cdot \vec{\varepsilon}_{2}+\frac{1}{4} \cdot \imath_{p-1}(v)\right) .
$$

This explicitly describes an embedding of $T^{p}=S_{1}^{1} \times \cdots \times S_{p}^{1}$ into $\mathbb{R}_{2, \ldots, p+2}^{p+1}$. In Figure 1, the images of $\imath_{p-1}$ and $\imath_{p}$ are schematically presented on the left and the right, respectively. One may imagine $\vec{\varepsilon}_{1}$ points perpendicularly outward the page. Observe that the image of $T^{p}$ is invariant under $\rho_{p}(u)$.

Definition 3.1. An embedding $\imath: T^{p} \hookrightarrow \mathbb{R}^{p+2}$ is said to be unknotted if there is a diffeomorphism $g: \mathbb{R}^{p+2} \rightarrow \mathbb{R}^{p+2}$ such that $\imath$ and $g \circ \imath_{p}$ have the same image, i.e., $\imath\left(T^{p}\right)=g \circ \imath_{p}\left(T^{p}\right)$.

Lemma 3.3. For any unknotted embedding $\imath: T^{p} \hookrightarrow \mathbb{R}^{p+2}$, the induced spin structure $\imath^{\sharp}\left(\varsigma^{p+2}\right)$ is not the Lie-group spin structure on $T^{p}$.

Proof. One can easily see that the standard embedding $\imath_{p}: T^{p}=S_{1}^{1} \times \cdots \times S_{p}^{1} \hookrightarrow \mathbb{R}^{p+2}$ can be extended to an embedding of $D^{2} \times T^{p-1}=D^{2} \times S_{2}^{1} \times \cdots \times S_{p}^{1}$ to $\mathbb{R}^{p+2}$, for $p \geq 1$, using an induction argument. Thus $\imath$ also has a Seifert hypersurface $\Sigma \subset \mathbb{R}^{p+2}$ diffeomorphic to $D^{2} \times T^{p-1}$. From the proof of Proposition 1.1, $\left(T^{p}, \imath^{\sharp}\left(\varsigma^{p+2}\right)\right)$ is the spin boundary of a spin structure on $\Sigma$. However, the spin structures on $\Sigma \cong D^{2} \times$ $T^{p-1}$ are $\varsigma^{2} \oplus \sigma$, where $\sigma \in \mathcal{S}\left(T^{p-1}\right)$, and these induce $\partial \varsigma^{2} \oplus \sigma$ on $\partial \Sigma \cong S^{1} \times T^{p-1}$, which disagree with the Lie-group spin structure along the loop $S^{1} \times *$.

Proof of Theorem 1.1. Lemma 3.1 proves $\left[\mathrm{MCG}_{\text {Top }}\left(T^{p}\right): \mathscr{E}_{\text {Top }}(\imath)\right] \geq 2^{p}-1$. To see that any unknotted embedding $\imath: T^{p} \hookrightarrow \mathbb{R}^{p+2}$ realizes the lower bound, note $\mathrm{MCG}_{\mathrm{Top}}\left(T^{p}\right)=$ $\mathscr{I}_{\text {Top }}\left(T^{p}\right) \rtimes \operatorname{Mod}\left(T^{p}\right)$. By Lemma $3.2, \mathscr{I}_{\text {Top }}\left(T^{p}\right) \leq \mathscr{E}_{\text {Top }}(\imath)$. On the other hand, [1, Theorem 1.4] showed $\operatorname{Mod}\left(T^{p}\right)$ (denoted as $\operatorname{Aut}\left(T^{p}\right)$ there) has a subgroup of index $2^{p}-1$, 

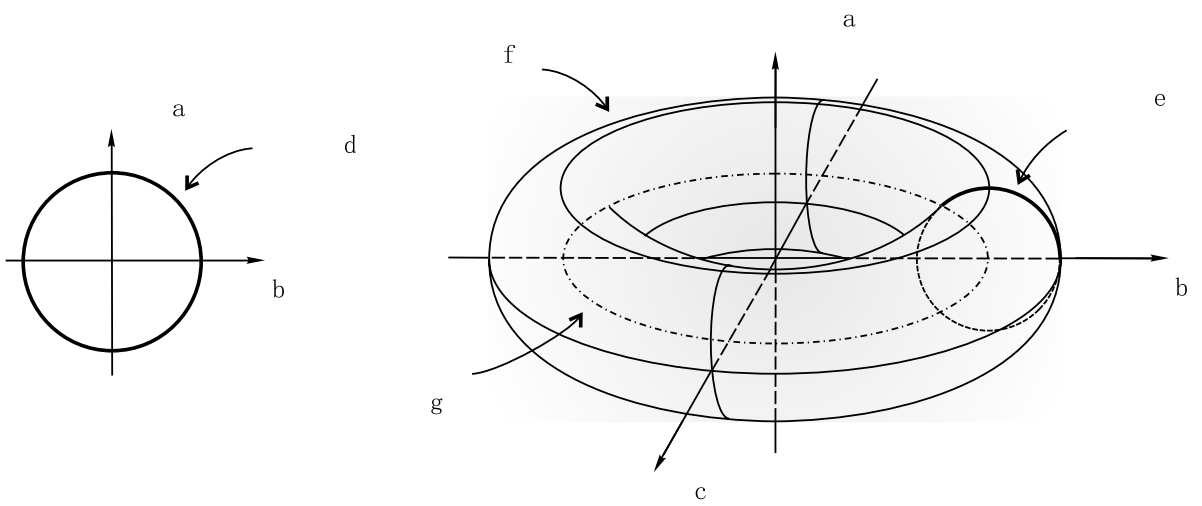

Figure 1. The standard model.

which is diffeomorphically extendable. Therefore, $\left[\mathrm{MCG}_{\mathrm{Top}}\left(T^{p}\right): \mathscr{E}_{\mathrm{Top}}(\imath)\right] \leq 2^{p}-1$, and hence the index is exactly $2^{p}-1$.

To prove Corollary 1.1, we need an elementary lemma in group theory.

Lemma 3.4. If $G$ is a subgroup of a semi-direct product of groups $N \rtimes H$, then $[N \rtimes H: G] \leq[N: N \cap G] \cdot[H: H \cap G]$.

Proof. Let $N^{\prime}=N \cap G, H^{\prime}=H \cap G$. Clearly $H^{\prime}$ preserves $N^{\prime}$ under the conjugation, so the subgroup $N^{\prime} H^{\prime}$ is also a semi-direct product. Note $\left[N H: N^{\prime} H^{\prime}\right]=[N H$ : $\left.N H^{\prime}\right] \cdot\left[N H^{\prime}: N^{\prime} H^{\prime}\right]$. As $N$ is normal in both $N H$ and $N H^{\prime}$, quotienting out $N$ yields $\left[N H: N H^{\prime}\right]=\left[H: H^{\prime}\right]$. Because $N \cap N^{\prime} H^{\prime}=N^{\prime}$ as $N^{\prime} H^{\prime}$ is a semi-direct product, the map $N \rightarrow N H^{\prime} / N^{\prime} H^{\prime}$ descends to a bijection $N / N^{\prime} \rightarrow N H^{\prime} / N^{\prime} H^{\prime}$ between the cosets, so $\left[N H^{\prime}: N^{\prime} H^{\prime}\right]=\left[N: N^{\prime}\right]$. Thus $[N H: G] \leq\left[N H: N^{\prime} H^{\prime}\right]=\left[N: N^{\prime}\right] \cdot[H:$ $\left.H^{\prime}\right]$.

Proof of Corollary 1.1. $\left[\operatorname{MCG}_{\mathcal{C}}\left(T^{p}\right): \mathscr{E}_{\mathcal{C}}(\imath)\right] \geq 2^{p}-1$ follows from Lemmas 3.1, 3.3. By Lemma 3.2, $\left[\mathscr{I}_{\mathcal{C}}\left(T^{p}\right): \mathscr{I}_{\mathcal{C}}\left(T^{p}\right) \cap \mathscr{E}_{\mathcal{C}}(\imath)\right]$ is finite. By $\left[1\right.$, Theorem 1.4], $\left[\operatorname{Mod}\left(T^{p}\right)\right.$ : $\left.\operatorname{Mod}\left(T^{p}\right) \cap \mathscr{E}_{\mathcal{C}}(\imath)\right]$ is finite. Therefore, as $\operatorname{MCG}_{\mathcal{C}}\left(T^{p}\right)=\mathscr{I}_{\mathcal{C}}\left(T^{p}\right) \rtimes \operatorname{Mod}\left(T^{p}\right)$, the index $\left[\operatorname{MCG}_{\mathcal{C}}\left(T^{p}\right): \mathscr{E}_{\mathcal{C}}(\imath)\right]$ is also finite by Lemma 3.4. Note clearly $\left[\operatorname{MCG}_{\mathcal{C}}\left(T^{p}\right): \mathscr{E}_{\mathcal{C}}(\imath)\right]=$ $2^{p}-1$ when $p \leq 3$.

\section{Embedded surfaces in $\mathbb{R}^{4}$}

In this section, we prove Theorem 1.2. Note $\operatorname{MCG}_{\mathcal{C}}\left(F_{g}\right)$ for $\mathcal{C}=\operatorname{Diff}, \mathrm{PL}, \mathrm{Top}$ are all canonically isomorphic to $\operatorname{Out}\left(\pi_{1}\left(F_{g}\right)\right)$ due to the Dehn-Nielsen-Baer theorem (cf. [11]).

Let $\mathcal{S}\left(F_{g}\right)$ be the space of spin structures on a closed connected oriented surface $F_{g}$ of genus $g$. There is a surjective map:

$$
\mathcal{S}\left(F_{g}\right) \stackrel{[\cdot]}{\longrightarrow} \Omega_{2}^{\text {Spin }} \stackrel{\text { Arf }}{\longrightarrow} \mathbb{Z}_{2},
$$

where $\Omega_{2}^{\text {Spin }}$ is the second spin cobordism group and Arf is the Arf isomorphism. More precisely, for any $\sigma \in \mathcal{S}\left(F_{g}\right)$, there is an associated non-singular quadratic function $q_{\sigma}: H_{1}\left(F_{g} ; \mathbb{Z}_{2}\right) \rightarrow \mathbb{Z}_{2}$, such that $q_{\sigma}(\alpha)=0$ (resp. 1) if the spin structure on $F_{g}$ 
restricted to the bounding (resp. Lie-group) spin structure on $\alpha$. Note $q_{\sigma}(\alpha+\beta)=$ $q_{\sigma}(\alpha)+q_{\sigma}(\beta)+\alpha \cdot \beta$ where $\alpha \cdot \beta$ is the $\mathbb{Z}_{2}$-intersection number, and $\sigma=\sigma^{\prime}$ if and only if $q_{\sigma}=q_{\sigma^{\prime}}$. Thus, $\operatorname{Arf}([\sigma])$ is defined as the Arf invariant of the non-singular quadratic form $q_{\sigma}$. Recall that for a non-singular quadratic form $q$ on $V \cong \mathbb{Z}_{2}^{\oplus 2 g}$, $\operatorname{Arf}(q)$ is 0 (resp. 1) if and only if $q$ vanishes on exactly $2^{2 g-1}+2^{g-1}$ (resp. $2^{2 g-1}-2^{g-1}$ ) elements, (cf. [12, Appendix]). Correspondingly, $\mathcal{S}\left(F_{g}\right)$ is a disjoint union:

$$
\mathcal{S}\left(F_{g}\right)=\mathcal{B}_{g} \sqcup \mathcal{U}_{g}
$$

of bounding and unbounding spin structures. We denote the cardinal numbers of $\mathcal{B}_{g}$, $\mathcal{U}_{g}$ as $b_{g}, u_{g}$, respectively.

Lemma 4.1. For $g \geq 1, b_{g}=2^{2 g-1}+2^{g-1}$ and $u_{g}=2^{2 g-1}-2^{g-1}$.

Proof. For $g=1$, it is well known that the only unbounding spin structure on $F_{1}=T^{2}$ is the Lie-group spin structure, so $b_{1}=3, u_{1}=1$. In general, any pair of two spin structures $\sigma_{g} \in \mathcal{S}\left(F_{g}\right), \delta \in \mathcal{S}\left(T^{2}\right)$ determines a bounding (resp. unbounding) spin structure on $F_{g+1} \cong F_{g} \# T^{2}$ if and only if $\operatorname{Arf}\left(\left[\sigma_{g}\right]\right)$ and $\operatorname{Arf}([\delta])$ have the same (resp. distinct) parity. This implies $b_{g+1}=b_{1} \times b_{g}+u_{1} \times u_{g}=3 b_{g}+u_{g}$, and $u_{g+1}=$ $b_{1} \times u_{g}+u_{1} \times b_{g}=3 u_{g}+b_{g}$, so $b_{g+1}-u_{g+1}=2\left(b_{g}-u_{g}\right)=\cdots=2^{g}\left(b_{1}-u_{1}\right)=2^{g+1}$. Using $b_{g}-u_{g}=2^{g}$ and $b_{g}+u_{g}=2^{2 g}$, we see $b_{g}=2^{2 g-1}+2^{g-1}, u_{g}=2^{2 g-1}-2^{g-1}$.

There is a natural action of $\operatorname{MCG}_{\mathcal{C}}\left(F_{g}\right)$ on $\mathcal{S}\left(F_{g}\right)$, where any $[\tau] \in \operatorname{MCG}_{\mathcal{C}}\left(F_{g}\right)$ acts as the pull-back $\tau^{*}: \mathcal{S}\left(F_{g}\right) \rightarrow \mathcal{S}\left(F_{g}\right)$.

Lemma 4.2. For $g \geq 1, \operatorname{MCG}_{\mathcal{C}}\left(F_{g}\right)$ acts invariantly and transitively on $\mathcal{B}_{g}$ and $\mathcal{U}_{g}$.

Proof. The invariance of the $\operatorname{MCG}_{\mathcal{C}}\left(F_{g}\right)$-action on $\mathcal{B}_{g}$ and $\mathcal{U}_{g}$ follows immediately from, for example, counting vanishing elements of the associated quadratic forms $q_{\sigma}, q_{\tau^{*}(\sigma)}$ for $\sigma \in \mathcal{S}\left(F_{g}\right)$ and $[\tau] \in \operatorname{MCG}_{\mathcal{C}}\left(F_{g}\right)$. It suffices to prove the transitivity of the action. We argue by induction on $g \geq 1$.

When $g=1, F_{1}$ is $T^{2} \cong S_{1}^{1} \times S_{2}^{1}$, and $\operatorname{MCG}_{\mathcal{C}}\left(T^{2}\right) \cong \operatorname{SL}(2, \mathbb{Z})$ is generated by the Dehn twists $D_{1}, D_{2}$ along the first and second factors. It is straightforward to check that $\operatorname{MCG}_{\mathcal{C}}\left(T^{2}\right)$ acts transitively on $\mathcal{B}_{1}$ and $\mathcal{U}_{1}$.

Suppose for some $g \geq 1, \operatorname{MCG}_{\mathcal{C}}\left(F_{g}\right)$ acts transitively on $\mathcal{B}_{g}$ and $\mathcal{U}_{g}$ for some $g \geq 1$. To see $\operatorname{MCG}_{\mathcal{C}}\left(F_{g+1}\right)$ acts transitively on $\mathcal{B}_{g+1}$, let $\sigma, \sigma^{\prime} \in \mathcal{B}_{g+1}$. Pick a connected sum decomposition $F_{g+1} \cong F_{g} \# T^{2}$, which induces a decomposition $H_{1}\left(F_{g+1} ; \mathbb{Z}_{2}\right) \cong$ $H_{1}\left(F_{g} ; \mathbb{Z}_{2}\right) \oplus H_{1}\left(T^{2} ; \mathbb{Z}_{2}\right)$. Then $\sigma$ determines spin structures $\sigma_{g} \in \mathcal{S}\left(F_{g}\right)$ and $\delta \in$ $\mathcal{S}\left(T^{2}\right)$ so that $[\sigma]=\left[\sigma_{g}\right]+[\delta]$ in $\Omega_{2}^{\text {Spin }}$, and similarly $\sigma^{\prime}$ determines $\sigma_{g}^{\prime}$, $\delta^{\prime}$ so that $\left[\sigma^{\prime}\right]=\left[\sigma_{g}^{\prime}\right]+\left[\delta^{\prime}\right]$. If $\left[\sigma_{g}\right]=\left[\sigma_{g}^{\prime}\right]$, and hence $[\delta]=\left[\delta^{\prime}\right]$, then by the induction assumption there are $\left[\tau_{g}\right] \in \operatorname{MCG}_{\mathcal{C}}\left(F_{g}\right)$ and $[\phi] \in \operatorname{MCG}_{\mathcal{C}}\left(T^{2}\right)$ such that $\tau_{g}^{*}\left(\sigma_{g}\right)=\sigma_{g}^{\prime}, \phi^{*}(\delta)=\delta^{\prime}$. Then one finds an element $[\tau] \in \operatorname{MCG}_{\mathcal{C}}\left(F_{g+1}\right)$, where $\tau=\tau_{g} \# \phi$, such that $\tau^{*}([\sigma])=\sigma^{\prime}$.

Now we consider the case if $\left[\sigma_{g}\right] \neq\left[\sigma_{g}^{\prime}\right]$, and hence $[\delta] \neq\left[\delta^{\prime}\right]$. Thus one of $\delta, \delta^{\prime} \in$ $\mathcal{S}\left(T^{2}\right)$ is the Lie-group spin structure, and the other is a spin-boundary, but there always exists some non-trivial $[\alpha] \in H_{1}\left(T^{2} ; \mathbb{Z}_{2}\right)$ such that $\left.\delta\right|_{\alpha}=\left.\delta^{\prime}\right|_{\alpha}$. For any $[\beta] \in$ $H_{1}\left(T^{2} ; \mathbb{Z}_{2}\right)$ with $\alpha \cdot \beta=1$, that $[\delta] \neq\left[\delta^{\prime}\right]$ implies $\left.\delta\right|_{\beta} \neq\left.\delta^{\prime}\right|_{\beta}$. On the other hand, there is some non-trivial $[\gamma] \in H_{1}\left(F_{g} ; \mathbb{Z}_{2}\right)$, such that $\left.\sigma_{g}\right|_{\gamma} \neq\left.\sigma_{g}^{\prime}\right|_{\gamma}$. Let $[\tilde{\beta}]=[\beta]+[\gamma] \in$ $H_{1}\left(F_{g+1} ; \mathbb{Z}_{2}\right)$, we have $\alpha \cdot \tilde{\beta}=1$, and the difference $\sigma-\sigma^{\prime} \in H^{1}\left(F_{g} ; \mathbb{Z}_{2}\right)$ vanishes on $[\alpha]$ and $[\tilde{\beta}]$. We may take two simple closed-curve representatives $\alpha, \tilde{\beta} \subset F_{g+1}$ such that $\alpha \cap \tilde{\beta}$ is a single point. A regular neighborhood of $\alpha \cup \tilde{\beta}$ on $F_{g+1}$ is a punctured torus 
$\tilde{T} \backslash *$, which gives another connected sum decomposition of $F_{g+1}=\tilde{F}_{g} \# \tilde{T}$. It is clear that with respect to this decomposition, the induced spin structures $\tilde{\sigma}_{g}, \tilde{\sigma}_{g}^{\prime} \in \mathcal{S}\left(F_{g}\right)$, $\tilde{\delta}, \tilde{\delta}^{\prime} \in \mathcal{S}\left(F_{g}\right)$ satisfy $\left[\tilde{\sigma}_{g}\right]=\left[\tilde{\sigma}_{g}^{\prime}\right],[\tilde{\delta}]=\left[\tilde{\delta}^{\prime}\right]$, so we apply the previous case to obtain some $[\tilde{\tau}] \in \mathrm{MCG}_{\mathcal{C}}\left(F_{g+1}\right)$, such that $\tilde{\tau}^{*}([\sigma])=\sigma^{\prime}$. This means $\mathrm{MCG}_{\mathcal{C}}\left(F_{g+1}\right)$ acts transitively on $\mathcal{B}_{g+1}$.

The proof for the transitivity of the $\operatorname{MCG}_{\mathcal{C}}\left(F_{g+1}\right)$-action on $\mathcal{U}_{g+1}$ is similar, so we complete the induction.

Proof of Theorem 1.2. Because $\mathrm{MCG}_{\mathrm{Top}}\left(F_{g}\right)$ are represented by self-diffeomorphisms, by Proposition 1.1, any element in $\mathscr{E}_{\text {Top }}(\imath)$ preserves $\imath^{\sharp}\left(\varsigma^{4}\right) \in \mathcal{B}_{g} \subset \mathcal{S}\left(F_{g}\right)$. On the other hand, $\mathrm{MCG}_{\mathrm{Top}}\left(F_{g}\right)$ acts transitively on $\mathcal{B}_{g}$ (Lemma 4.2$)$. Therefore, $\left[\mathrm{MCG}_{\mathrm{Top}}\left(F_{g}\right)\right.$ : $\left.\mathscr{E}_{\mathrm{Top}}(\imath)\right] \geq\left|\mathcal{B}_{g}\right|=2^{2 g-1}+2^{g-1},($ Lemma 4.1$)$.

Corollary 1.2 is an easy consequence of Lemma 4.2 :

Proof of Corollary 1.2. Observe that the action of $\mathrm{MCG}_{\mathcal{C}}\left(F_{g}\right)$ on $\mathcal{S}\left(F_{g}\right)$ descends to an action of a group $\Gamma<\operatorname{Aut}^{+}\left(H_{1}\left(F_{g} ; \mathbb{Z}_{2}\right)\right)$ : indeed, if $\tau$ projects to the identity of $\operatorname{Aut}^{+}\left(H_{1}\left(F_{g} ; \mathbb{Z}_{2}\right)\right), q_{\tau^{*} \sigma}([\alpha])=q_{\sigma}\left(\tau_{*}[\alpha]\right)=q_{\sigma}([\alpha])$, for any $[\alpha] \in H_{1}\left(F_{g} ; \mathbb{Z}_{2}\right)$, so $\tau^{*} \sigma=\sigma$ for any $\sigma \in \mathcal{S}\left(F_{g}\right)$. $\Gamma$ is a finite group isomorphic to $\operatorname{Sp}\left(2 g, \mathbb{Z}_{2}\right)$ as it preserves the $\mathbb{Z}_{2}$-intersection form. Then Lemma 4.2 implies $\Gamma$ acts transitively on $\mathcal{B}_{g}$, so for any $\sigma \in \mathcal{B}_{g}$, $\operatorname{Stab}_{\Gamma}(\sigma)<\Gamma$ has index $b_{g}=2^{2 g-1}+2^{g-1}$. Since id $\in \operatorname{Stab}_{\Gamma}(\sigma)$ for all $\sigma \in \mathcal{B}_{g}$, the subset:

$$
W=\bigcup_{\sigma \in \mathcal{B}_{g}} \operatorname{Stab}_{\Gamma}(\sigma) \subset \Gamma,
$$

has at most $b_{g}\left(\frac{|\Gamma|}{b_{g}}-1\right)+1<|\Gamma|$ elements. Thus for any $[\tau] \in \Gamma \backslash W, \tau$ does not fix any $\sigma \in \mathcal{B}_{g}$. In particular, for any smooth embedding $\imath: F_{g} \hookrightarrow \mathbb{R}^{4}, \imath^{\sharp}(\varsigma) \in \mathcal{B}_{g}$ will not be invariant under $\tau$. By Proposition 1.1, $[\tau] \notin \mathscr{E}_{\text {Top }}(\imath)$.

\section{Acknowledgments}

The authors thank Robert Edwards, Andrey Gogolev, Jonathan Hillman, Robion Kirby, and Hongbin Sun for helpful communications. The authors also thank the referee for valuable comments. Ding and Wang are partially supported by grant no. 10631060 of the National Natural Science Foundation of China.

\section{References}

[1] F. Ding, Y. Liu, S.-C. Wang and J.-G. Yao, Extending $T^{p}$ automorphisms over $\mathbb{R}^{p+2}$ and realizing DE attractors, Discrete Contin. Dyn. Syst. 32(5) (2012), 1639-1655.

[2] D. Erle, Quadratische Formen als Invarianten von Einbettungen der Kodimension 2, (German), Topology 8 (1969), 99-114.

[3] M. Freedman, F. Quinn, Topology of 4-manifolds, Princeton Mathematical Series, 39, Princeton University Press, 1990.

[4] L. Guillou and A. Marin, Une extension d'un théorème de Rohlin sur la signature, (French), [An extension of Rokhlin's signature theorem] à la recherche de la topologie perdue, 62, 97-118, Progr. Math., Birkhäuser Boston, Boston, MA, 1986.

[5] A. Hatcher, Homeomorphisms of sufficiently large $P^{2}$-irreducible 3-manifolds, Topology 15 (1976), 343-347.

[6] A. Hatcher, Concordance spaces, higher simple-homotopy theory, and applications, Algebraic and geometric topology Part 1, 3-21, Proc. Symp. Pure Math. XXXII, Amer. Math. Soc., Providence, RI, 1978. 
[7] A. Hatcher, A proof of the Smale conjecture, Ann. Math. 117 (1983), 553-607.

[8] S. Hirose, On diffeomorphisms over surfaces trivially embedded in the 4-sphere, Algebr. Geom. Topol. 2 (2002), 791-824.

[9] M. W. Hirsch, Differential topology, Springer-Verlag, New York-Heidelberg, 1976.

[10] W.-C. Hsiang and R. W. Sharpe, Parametrized surgery and isotopy, Pacific J. Math. 67(2) (1976), 401-459.

[11] N. V. Ivanov, Mapping class groups, Handbook of Geometric Topology, North-Holland, Amsterdam, 2002.

[12] R. C. Kirby, The topology of 4-manifolds, Lecture Notes in Math. 1374, Springer-Verlag, Berlin, 1990.

[13] R. C. Kirby and L. C. Siebenmann, Normal bundles for codimension 2 locally flat imbeddings, (Proc. Conf., Park City, Utah, 1974), pp. 310-324, Lecture Notes Math. 438, Springer, Berlin, 1975.

[14] R. C. Kirby and L. R. Taylor, Pin structures on low-dimensional manifolds, in Geometry of Low-Dimensional Manifolds, Vol. 2 (Durham, 1989), 177-242, Cambridge University Press, Cambridge, 1990.

[15] J. Milnor, Spin structures on manifolds, Enseign. Math. (2) 9 (1963), 198-203.

[16] J. Milnor and J. Stasheff, Characteristic classes, Annals of Mathematics Studies 76, Princeton University Press, Princeton, NJ; University of Tokyo Press, Tokyo, 1974.

[17] J. M. Montesinos, On twins in the four-sphere, I., Quart. J. Math. Oxford Ser. (2) 34(134) (1983), 171-199.

[18] V. A. Rohlin, Proof of a conjecture of Gudkov, Funct. Anal. Appl. 6 (1972), 136-138.

[19] O. Saeki, A. Szücs and M. Takase, Regular homotopy classes of immersions of 3-manifolds into 5-space, Manuscripta Math. 108(1) (2002), 13-32.

[20] W.-T. Wu, Classes caractéristiques et i-carrés d'une variété, (French), C. R. Acad. Sci. Paris 230 (1950), 508-511.

School of Mathematical Sciences, Peking University, Beijing 100871, People's Republic OF CHINA

E-mail address: dingfan@math.pku.edu.cn

Department of Mathematics, 970 Evans Hall, University of California Berkeley, CA 94720-3840, USA

E-mail address: yliu@math.berkeley.edu

School of Mathematical Sciences, Peking University, Beijing 100871, People's Republic OF CHINA

E-mail address: wangsc@math.pku.edu.cn

Department of Mathematics, 970 Evans Hall, University of California, Berkeley, CA 94720-3840, USA

E-mail address: jiangangyao@gmail.com 
